\title{
放牧对草地生态系统服务和功能权衡关系的影响
}

\author{
汤永康 $^{1,2}$ 武艳涛 ${ }^{1}$ 武 鬼 ${ }^{3}$ 郭之伟 $^{1}$ 梁存柱 $^{*}$ 王敏杰 $^{1}$ 常佩静 $^{2}$
}

${ }^{1}$ 内蒙古大学生态与环境学院, 呼和浩特 $010021 ;{ }^{2}$ 阿拉善盟气象局, 内蒙古阿拉善 $750300 ;{ }^{3}$ 内蒙古自治区锡林浩特国家气候观象台, 内蒙古锡林浩特 026000

摘 要 内蒙古草原是我国北方的重要生态屏障和绿色畜牧业基地, 放牧是草原生态系统的主要利用和管理方式, 在放牧管 理中充分发挥生态系统某一项或几项服务和功能最大利用价值时, 往往会与其他服务(功能)发生冲突, 需要权衡多项生态系 统服务和功能, 制定合理的放牧管理制度。该研究以内蒙古锡林郭勒典型草原为例, 通过设置不放牧、轻度放牧、中度放牧 以及重度放牧的放牧梯度, 从多项生态系统服务和功能权衡的角度比较了最适放牧管理强度。结果显示, 在放牧管理的草地 生态系统服务和功能的权衡中, 权衡、协同、不相关关系同时存在, 如土壤呼吸速率与植物群落净生长量、生物多样性与植 物群落净光合速率表现为权衡关系, 植物群落净生长量分别与土壤含水量、植物群落净光合速率及草地蒸散速率存在协同关 系, 土壤有机碳含量与其他服务或功能间呈不相关关系; 放牧能不同程度地削弱多项生态系统服务及功能间的权衡关系(冲 突对立关系); 中度放牧条件下的多项生态系统服务及功能的协同性最佳。

关键词 生态系统服务; 生态系统功能; 权衡分析; 典型草原; 放牧梯度

汤永康, 武艳涛, 武魁, 郭之伟, 梁存柱, 王敏杰, 常佩静 (2019). 放牧对草地生态系统服务和功能权衡关系的影响. 植物生态学报, 43, 408-417. DOI: $10.17521 /$ cjpe.2018.0289

\section{Changes in trade-offs of grassland ecosystem services and functions under different grazing intensities}

TANG Yong-Kang ${ }^{1,2}$, WU Yan-Tao ${ }^{1}$, WU Kui ${ }^{3}$, GUO Zhi-Wei ${ }^{1}$, LIANG Cun-Zhu ${ }^{1 *}$, WANG Min-Jie ${ }^{1}$, and CHANG Pei-Jing ${ }^{2}$

${ }^{1}$ Faculty of Ecology and Environment, Inner Mongolia University, Hohhot 010021, China; ${ }^{2}$ Alxa League Meteorological Bureau, Alxa, Nei Mongol 750300, China; and ${ }^{3}$ Xilinhot National Climate Observing Station, Xilinhot, Nei Mongol 026000, China

\section{Abstract}

Aims The Nei Mongol Steppe plays an important role for livestock production, and it has been one of the green ecological shelters for Northern China. Enhancing some ecosystem services (provisioning services) in managing the ecosystems may causes reductions of other services, such as regulating and supporting services. The knowledge on how grazing intensity influence the trade-offs is lacking.

Methods In order to find out the optimized grazing regimes, we conducted an experiment on a typical steppe in Xinlin Gol with four grazing intensities (no grazing, light grazing, moderate grazing and heavy grazing) in the Nei Mongol Steppe.

Important findings Our results showed that: synergetic, trade-offs and no relationships among different ecosystem services existed in all treatments on the managed steppe system. The trade-offs relationship was found between soil respiration rate and net plant biomass growth, and between biodiversity and net photosynthetic rate. However the synergetic relationships were observed between net plant biomass growth and soil water content, between net plant growth biomass and net photosynthetic rate, and also between grassland evapotranspiration rate and biodiversity. The results indicated soil organic carbon was not related to other ecosystem services and functions. Grazing could weaken the conflicts among the ecosystem services. Moderate grazing intensity maximumize ecosystem services and functions.

Key words ecosystem service; ecosystem function; trade-off analysis; typical steppe; grazing intensity

Tang YK, Wu YT, Wu K, Guo ZW, Liang CZ, Wang MJ, Chang PJ (2019). Changes in the trade-offs of grassland ecosystem services and functions under different grazing intensities. Chinese Journal of Plant Ecology, 43, 408-417. DOI: 10.17521/cjpe.2018.0289

收稿日期Received: 2018-11-14 接受日期Accepted: 2019-05-05

基金项目：国家重点研发计划(2016YFC0500503)和内蒙古自治区科技计划(20140409)。Supported by the National Key R\&D Program of China (2016YFC0500503), and the Science and Technology Project of Nei Mongol Autonomous Region (20140409).

* 通信作者Corresponding author (bilcz@imu.edu.cn) 
生态系统服务是指人类从生态系统中获得的全 部利益, 包括支持服务、供给服务、调节服务、文 化服务(Millennium Ecosystem Assessment, 2005)。生 态系统功能是生态系统的固有属性, 主要包括物质 循环、能量流动和信息传递三大基本功能(Odum, 1971)。正常的生态系统通常具备同时维持多重生态 系统服务和生态系统功能的多功能性(Hector \& Bagchi，2007)。生态系统的多功能性导致人类利用 生态系统提供的服务及管理生态系统时需要考虑生 态系统不同功能之间的权衡与协同关系(Zavaleta et al., 2010; 徐炜等, 2016)。

由于生态系统类型的多样化及其相互关系的复 杂性, 过于强调某项生态系统服务时, 可能导致其 他服务被低估。当生态系统中某种服务或功能被大 量使用可能造成其他服务和功能的下降, 这种此消 彼长的对立冲突关系也称为权衡关系(Rodríguez et al., 2006); 与权衡对立的关系则称为协同, 是两种 及以上生态系统服务或功能同增或同减时的关系; 还有一种关系介于权衡与协同之间, 是指一种服务 或功能的改变对其他服务和功能的作用很微弱或无 差别的不相关关系(Naidoo et al., 2008)。不同利用方 式下生态系统服务和功能之间权衡/协同关系各异, 这推动了生态系统服务和功能的相互作用及权衡管 理决策研究(傅伯杰等, 2009)。

草地不仅能为人类提供具有直接经济价值的产 品, 还具有调节气候、维系生物基因库、保持水土、 固定 $\mathrm{CO}_{2}$ 和维持生物多样性等多种生态系统服务功 能(白永飞等, 2014)。草地生态系统中放牧引发各服 务及功能的权衡与协同关系变化是当前草地生态学 及草地管理亟待解决的重要科学问题(王德利和王 玲, 2019), 受到国内外学者的广泛关注。Davidson 等(2017)基于meta分析法研究了放牧对北美和欧洲 盐碱滩草地21项生态系统服务和功能指标的影响, 发现放牧会增加土壤容重和物种丰富度, 但会降低 地上生物量、冠层高度和调落物量。Austrheim等 (2016)通过在挪威南部的高寒草地放牧实验中得出 了维持载畜量 $\left(25 \mathrm{sheep} \cdot \mathrm{km}^{-2}\right)$ 时, 支持服务(植物生 物量、氮循环)、调节服务(栖息地开放性、土壤碳 存储量)、供给服务(薪柴)和无脊椎动物生物多样性 均高于减少载畜量 $\left(0\right.$ sheep $\left.\cdot \mathrm{km}^{-2}\right)$ 和增加载畜量 (80 sheep $\cdot \mathrm{km}^{-2}$ )处理的结论。Pan等(2014)对青藏高 原高寒草地的研究中权衡了肉产品供给服务和牧草
供给服务、草地碳调节服务的关系, 发现随着载畜 量不断增加, 羊肉的供给服务提高了2倍, 但伴随草 地净初级生产力供给服务和生态系统碳固定、保水 能力调节服务的骤降。Wang等(2019)对植物生物量 (地上、地下)、植物元素含量、昆虫多样性、土壤 养分循环、植物-微生物共生等12项指标在同等放牧 压力但不同畜种(牛单牧、羊单牧、牛羊混牧)的放 牧条件下进行标准化计算, 得出了混合放牧能显著 提高生物(植物、动物、微生物、土壤线虫等)多样 性指数和生态系统多功能性指数, 混合放牧下生物 多样性与生态系统多功能性的联系也比单一畜种下 各功能的联系更加紧密的结论。

内蒙古是我国北方重要的天然生态屏障和畜牧 业生产基地, 放牧是草原生态系统的主要利用方式 和管理方法。由于人们片面追求经济利益, 长期过 度放牧导致严重的草地退化, 不利于草地生态系统 可持续利用(汪诗平等, 1999)。众多文献从生物量、 物种多样性和家畜增长等单一生态系统服务或功能 讨论最适草地放牧管理方式(Mcintyre et al., 2003; Pucheta et al., 2010), 但不同的管理需求得出的最适 管理方式不同, 并且单一生态系统服务或功能的评 估过于片面, 无法从生态系统多功能性及权衡的角 度解释放牧管理方式是否合理。本实验以内蒙古典 型草原为研究对象, 基于不同放牧强度的草地管理 方式, 探究草地生态系统多项服务和功能的权衡关 系, 并以提高多项草地生态系统服务和功能的协同 性为目标, 合理地评价草地放牧管理方式, 为提高 草地整体服务能力, 优化家庭牧场草地资源管理和 实现草地可持续利用提供理论依据。

\section{1 材料和方法}

研究区位于内蒙古锡林浩特国家气候观象台内 牧业气象实验平台 $\left(44.14^{\circ} \mathrm{N}, 116.31^{\circ} \mathrm{E}\right)$, 属典型大 陆型气候, 气候干旱。根据2016和2017年两年的气 象观测记录, 平均年降水量 $240.2 \mathrm{~mm}$, 主要集中在 6-9月, 超过年降水量的 $70 \%$, 年平均气温 $4.1{ }^{\circ} \mathrm{C}$, 最热月平均气温 $24.1{ }^{\circ} \mathrm{C}$, 最冷月平均气温 $-19.7{ }^{\circ} \mathrm{C}$ (图1)。

实验区优势植被类型为大针茅(Stipa grandis) + 羊草(Leymus chinensis)群落, 主要组成物种包括多 年生禾草粘隐子草(Cleistogenes squarrosa)、根茎冰 草(Agropyron michnoi), 百合科的知母(Anemarrhena

DOI: $10.17521 /$ cjpe.2018.0289 


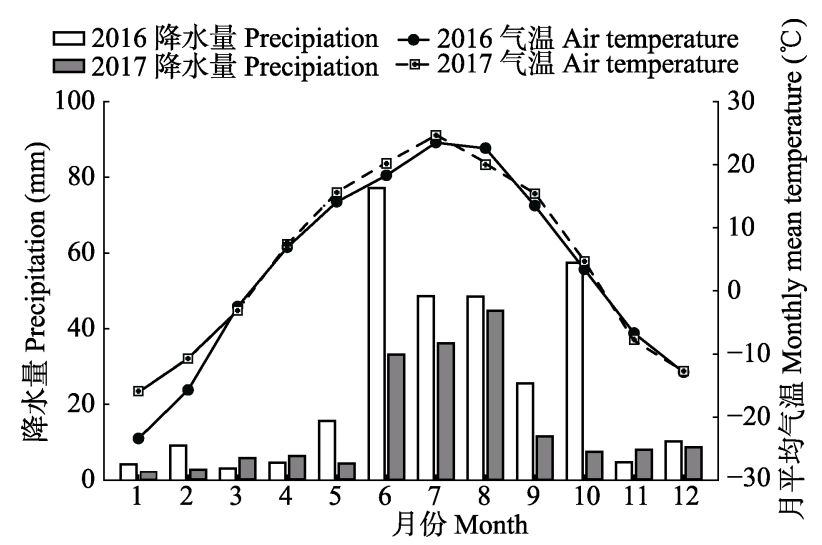

图1 2016-2017年锡林浩特市月平均气温与降水量分布情 况。

Fig. 1 An illustration of monthly mean air temperature and precipitation of Xilinhot from 2016 to 2017.

asphodeloides)、黄花非(Allium condensatum)、蒙古 非(Allium mongolicum), 豆科的乳白黄耆(Astragalus galactites)、多叶棘豆(Oxytropis myriophylla), 以及 莎草科的黄囊薹草(Carex korshinskyi)(梁茂伟等, 2016)。

\section{2 试验设计}

观象台放牧管理实验始于2013年6月，每年6-9 月放牧, 9月底结束当年放牧, 每月放牧一轮, 每年 往复进行放牧实验。样地设计采用随机区组, 共 12 块轮牧放牧样地, 分 3 组重复, 每组重复分 4 种处理 方式, 依次设置为围封不放牧 $(\mathrm{CK})$ 、轻度放牧 $(\mathrm{LG})$ 、 中度放牧 $(\mathrm{MG})$ 和重度放牧( $\mathrm{HG})$ 。每轮放牧开始时, 将 84 只健康的成年羯羊平均分为 3 组分别在 3 个 $\mathrm{LG}$ 样地放牧 1 天, 而后在MG样地放牧 1 天, 再到 $\mathrm{HG}$ 样 地放牧 1 天, 如此往复轮牧。待 $\mathrm{LG}$ 样地放牧达到 3 天 后, 即放牧样地共经过 9 天轮牧后, 再持续MG放牧 1 天、 $\mathrm{HG}$ 放牧 1 天的循环中。再经过 6 天放牧后, 即 $M G$ 放牧天数达 6 天后, 仅在 HG放牧 6 天。直至LG样 地放牧 3 天, $\mathrm{MG}$ 样地放牧 6 天, $\mathrm{HG}$ 样地放牧 12 天。连 续放牧 21 天后, 当月放牧结束, 当月的其余时间休 牧。小区面积 $120 \mathrm{~m} \times 120 \mathrm{~m}$, 小区间用网围栏分 割。7:00出牧, 中午对家畜补充饮水, 18:00收牧, 不 对家畜补饲。

\section{3 取样和计算方法}

\subsection{1 植物群落净生长量和生物多样性}

6月放牧前, 测定各实验样地地上、地下生物量。 地上生物量获取使用刚割方法, 随机选 5 个 $1 \mathrm{~m} \times 1 \mathrm{~m}$ 样方, 间隔 $20 \mathrm{~m}$, 记录物种的高度、丛幅、盖度和 株从数, 分种划割。6-9月当月放牧结束后采用同样
方法在各样地随机选 5 个样方分种刚割。围封样地每 10 天收集一次生物量。LG、MG和HG样地随机设置 3 个 $1 \mathrm{~m} \times 1 \mathrm{~m} \times 1.2 \mathrm{~m}$ 的铁笼, 每月牧后分种获取笼 内笼外地上生物量, 并移动铁笼。获取的植物在 $65{ }^{\circ} \mathrm{C}$ 下烘干至恒质量, 并称取质量。除6月外, 其他 各月牧前生物量以上月牧后生物量为准。植物群落 净生长量采用围笼法计算, 若放牧条件下群落净生 长量显著高于 $\mathrm{CK}$, 表现为超补偿生长; 若与 $\mathrm{CK}$ 无 差异, 呈等补偿生长; 若显著低于 $\mathrm{CK}$, 则为欠补偿 生长(韩国栋等, 1999; 薛睿等, 2010)。

生物多样性采用Shannon-Wiener指数 $\left(H^{\prime}\right)$ 表示:

$$
H^{\prime}=-\sum P_{i} \times \log _{2} P_{i}
$$

式中, $P_{i}$ 为第 $i$ 种植物生物量。

\subsection{2 土样有机碳含量}

9月放牧结束后, 在原笼外刈割样方内, 使用直 径为 $7 \mathrm{~cm}$ 的土钻, 在刚割后的样方中央钻取土样, 深度依次为 $0-10 、 10-20 、 20-30 \mathrm{~cm}$, 每样地 5 次重 复。使用油浴重铭酸钾滴定法计算土壤有机碳含量。

\subsection{3 土壤呼吸速率}

每月休牧期间, 选择晴朗、无风、地面较干燥 的 9:00-11:00, 使用 LI-6400-09 土壤呼吸叶室 (LI-COR, Lincoln, USA) 测定土壤呼吸速率 $\left(\mu \mathrm{mol} \cdot \mathrm{m}^{-2} \cdot \mathrm{s}^{-1}\right)$ 。每个样点设定循环测量 3 次, 每次循 环记录一个均值, 每个样地 4 次重复, 共测定 4 次。

\subsection{4 土壤含水量}

在8-9月雨后5-7天内, 使用直径7 $\mathrm{cm}$ 土钻, 钻 取表层0-10 cm 鲜土, 用塑封带装取密封, 使用烘干 法计算土壤含水量以评估不同放牧强度下土壤保水 能力, 共测定 3 次。

\subsection{5 植物群落净光合速率和草地蒸散速率}

每月休牧期间, 在晴朗无云的9:00-11:00, 采用 LI-6400-13标准叶室连接自制的静态同化箱测定植 物群落净光合速率和草地蒸散速率。静态同化箱由 $0.5 \mathrm{~m} \times 0.5 \mathrm{~m} \times 0.6 \mathrm{~m}$ 透明光罩和地框组成, 光罩内 壁对角线安装一对 $0.1 \mathrm{~m} \times 0.1 \mathrm{~m}$ 风扇保证内部环境 接近自然状态。设定测定时间 $2 \mathrm{~min}$, 每 $1 \mathrm{~s}$ 自动记录 一次净光合速率值 $\left(P_{\mathrm{n}}, \mu \mathrm{mol} \cdot \mathrm{m}^{-2} \cdot \mathrm{s}^{-1}\right)$ 和蒸散速率值 $\left(T_{\mathrm{r}}, \mathrm{mmol} \cdot \mathrm{m}^{-2} \cdot \mathrm{s}^{-1}\right)$, 每个样地 2 次重复, 共测定 4 次。

本实验中群落净光合速率用单位体积内 $\mathrm{CO}_{2}$ 被 吸收的速率表示, 即 $\mathrm{CO}_{2}$ 浓度下降的速率越快, 植 物群落净光合的同化能力越强, $\mathrm{CO}_{2}$ 被吸收的速率 值离横坐标轴越远, 就表示群落净光合速率越高。 


\subsection{6 生态系统服务及功能权衡关系}

对各生态系统服务和功能进行无量纲化处理, 处理后的生态系统服务(功能)系数范围在0-1 (图 2)。参与权衡的生态系统服务或功能作为横纵坐标 轴, 标准化处理后的生态系统服务或功能系数作为 横纵坐标 $(x, y)$, 如B点坐标为 $\left(x_{\mathrm{ES}-\mathrm{B} 1}, y_{\mathrm{ES}-\mathrm{B} 2}\right)$ 。以红色 的权衡平衡线(no trade-off)将坐标系均分成两部分, 均方根偏差 $(R M S D)$ 为坐标中点偏离平衡线的垂直 距离, 以此作为两生态系统服务(功能)的权衡关系, 距离越远，权衡关系越强烈，反之，权衡关系越弱, 协同关系越强。图示中 $\mathrm{B} 、 \mathrm{C}$ 点距离平衡线的垂直距 离相等, 并分别在平衡线两侧, 表示 $\mathrm{B}$ 更有利于 ES-2, 而C更有利于ES-1; A、B 点均在平衡线左侧, 表示二者均倾向于ES-2, 但B距平衡线更远, 说明B 点的权衡关系较A大。本研究选取2017年群落净生 长量、Shannon-Wiener多样性指数、土壤含水量、 草地蒸散速率、土壤有机碳含量、土壤呼吸速率和 群落净光合速率7项服务和功能参与草地生态系统 权衡和评估。

生态系统服务及功能权衡的计算:

$$
\begin{aligned}
& \widehat{E S}=\left(X-X_{\mathrm{Min}}\right) /\left(X_{\mathrm{Max}}-X_{\mathrm{Min}}\right) \\
& R M S D=\sqrt{\frac{1}{n-1} \sum_{i=1}^{n}\left(E S_{i}-\widehat{E S}\right)}
\end{aligned}
$$

标准化后生态系统服务或功能值 $\widehat{E S}$ 在 $(0,1)$ 之 间。 $X$ 为不同放牧强度下 3 组重复的均值, $X_{\mathrm{Min}}$ 和 $X_{\mathrm{Max}}$ 分别代表处理均值的极小值和极大值, $R M S D$ 为均

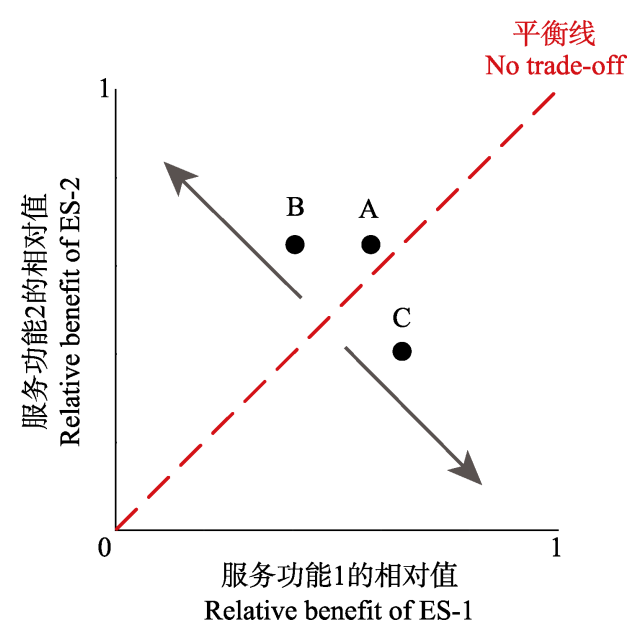

图2 生态系统服务和功能权衡关系图(Bradford \& D'Amato, 2012; Lu et al., 2014)。

Fig. 2 An illustration of trade-offs between ecosystem services (ES)(Bradford \& D'Amato, 2012; Lu et al., 2014).
方根偏差，表示生态系统服务和功能权衡关系的大 小(Pan et al., 2013; Lu et al., 2014)。

\section{4 数据处理与分析}

分析中放牧对各测量指标的单因素方差分析在 SPSS 17.0中进行计算, 单因素方差分析使用最小显 著差异 $(L S D)$ 法比较, $p<0.05$ 为差异显著。

\section{2 结果和分析}

\section{1 不同放牧强度下草地生态系统服务和功能的 变化}

草地生态系统供给服务中, 植物群落净生长量 LG显著高于 $\mathrm{CK}(p<0.05), \mathrm{MG}$ 和 $\mathrm{HG}$ 生长量与 $\mathrm{CK}$ 无 显著差异 $(p>0.05)$, 说明轻度放牧下植物群落呈超 补偿生长, 而中度和重度放牧下群落表现为等补偿 生长(图3A)。

草地生态系统调节、支持服务和功能中, 多样 性指数随放牧强度的增加呈显著下降变化 $(p<0.05)$; 土壤含水量和草地蒸散速率变化均与放牧强度呈负 相关变化，且CK与 $\mathrm{HG}$ 之间差异显著 $(p<0.05), \mathrm{LG}$ 、 $M G$ 与其他放牧处理间无明显差异 $(p>0.05)$; 土壤 有机碳含量 $\mathrm{CK}$ 显著大于 $\mathrm{MG}(p<0.05)$; 土壤呼吸 速率随放牧强度的增加呈下降变化，各放牧强度间 差异显著 $(p<0.05)$; 植物群落净光合速率呈先升后 降的变化，各放牧处理间无显著差异 $(p>0.05)$ (图 $3 \mathrm{~B}-3 \mathrm{G})$ 。

\section{2 草地多项生态系统服务和功能权衡关系对不} 同放牧强度的响应

\subsection{1 两项生态系统服务和功能在不同放牧强度 下的权衡}

在两项生态系统服务或功能的权衡关系中，与 不放牧相比，一些服务或功能权衡关系因放牧而增 大，如土壤呼吸速率与群落净生长量、多样性指数 与群落净光合速率，这使得自然状态下在权衡平衡 线附近的服务因放牧而更加偏离平衡线; 相对地, 有一些服务或功能的权衡关系因放牧而减小，如群 落净生长量分别与土壤含水量、群落净光合速率、 草地蒸散速率及多样性指数, 土壤呼吸速率分别与 草地蒸散速率及多样性指数, 这些在自然状态下距 离平衡线较远的服务和功能反而因放牧减小了权衡 关系; 还有一些服务或功能的关系并没有因为干扰 程度的改变而发生剧烈变化，如土壤有机碳含量分 别与群落净生长量、土壤含水量、群落净光合速率 
及多样性指数, 草地蒸散速率分别与多样性指数及 群落净光合速率等生态系统服务和功能的关系 (图4)。

\subsection{2 多项生态系统服务及功能的权衡}

不同放牧强度下, $R M S D$ 的大小关系为 $\mathrm{MG}<$
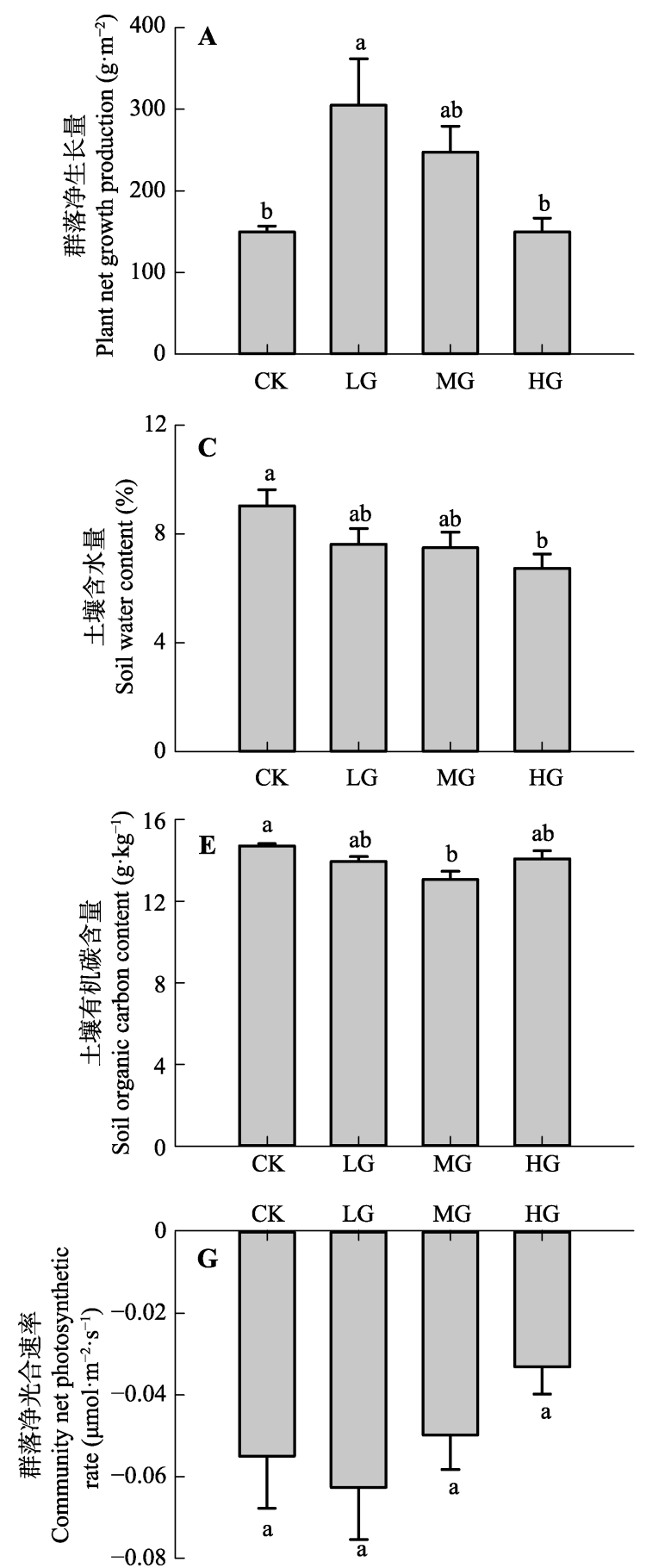

$\mathrm{HG}<\mathrm{LG}<\mathrm{CK}$ ，因此 $\mathrm{MG}$ 的多项生态系统服务和功 能权衡关系是最小的 $(R M S D=0.083)$, 生态系统因 放牧而削弱了各服务功能的权衡关系; 相对地, CK 的多项生态系统服务及功能权衡关系最大，各服务 间协同性较差，各服务功能之间竞争激烈(图5A)。
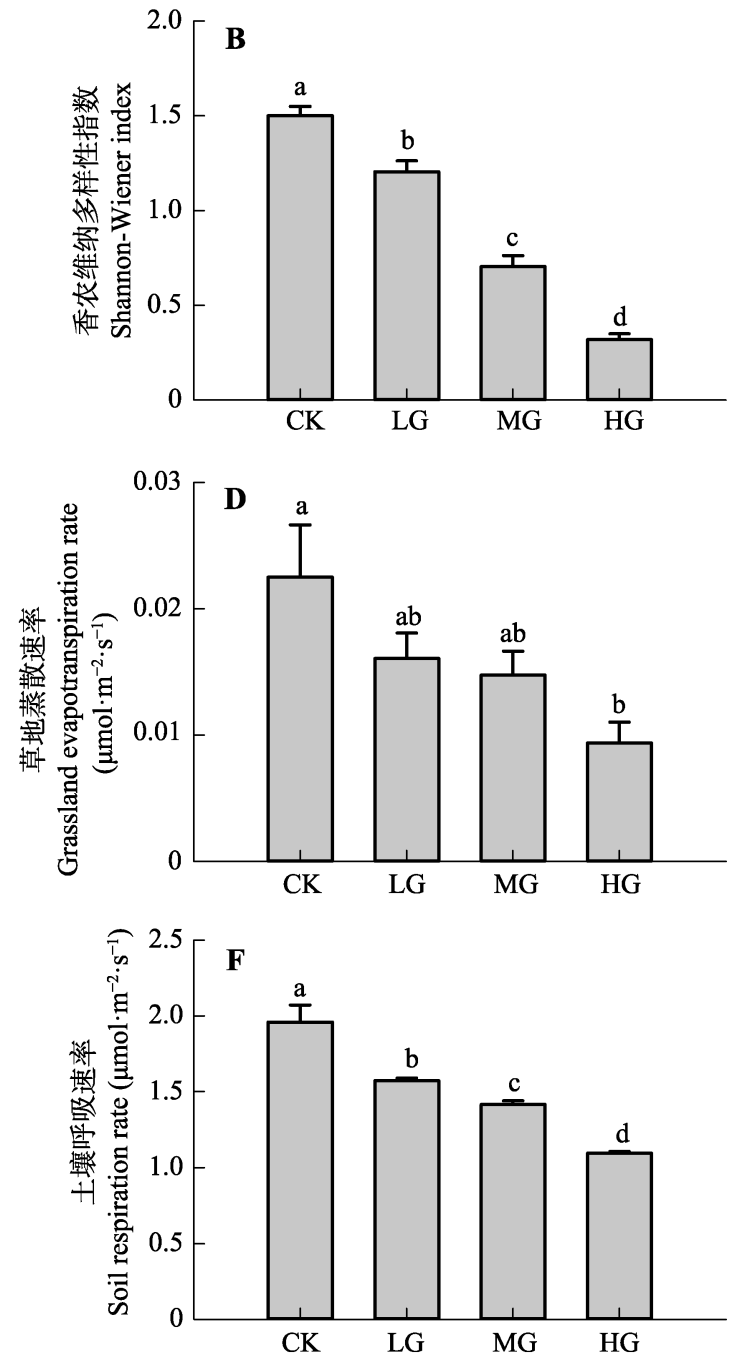

图3 不同放牧强度下草地生态系统服务和功能的变化(平均值+标准误差)。相同小写字母表示不同放牧强度下无显著差异 $(p>0.05)$ 。CK, 对照; LG, 轻度放牧; MG, 中度放牧; HG, 重度放牧。

Fig. 3 Variations of the ecosystem services (functions) of the studied grassland under different grazing intensities (mean $+S E$ ). The same lowercase letter means no significant different between grazing intensities ( $p>0.05)$. CK, control; LG, light grazing; MG, moderate grazing; $\mathrm{HG}$, heavy grazing. 


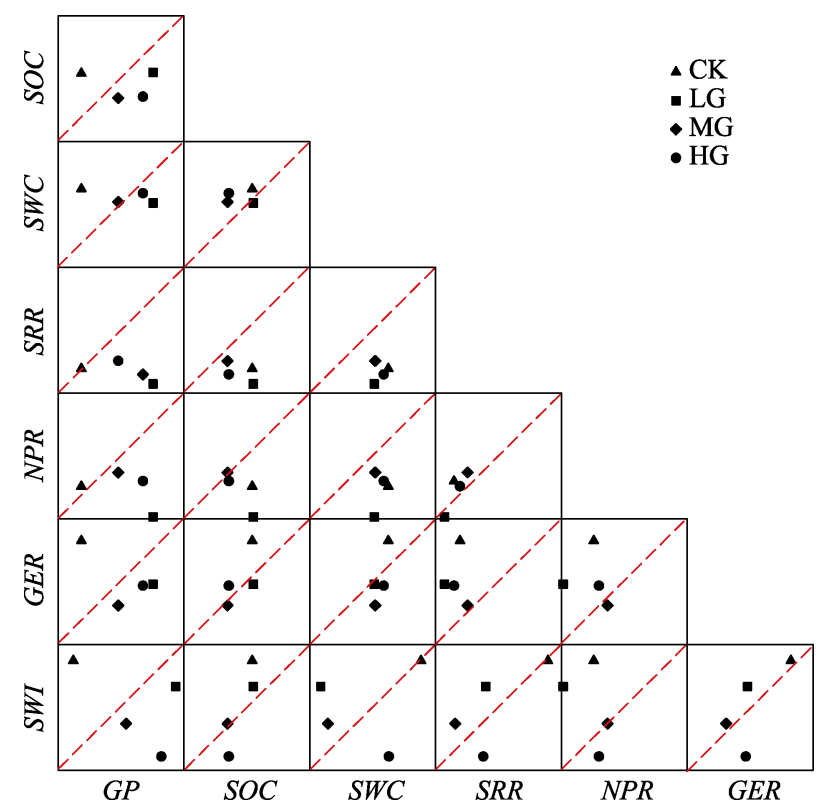

图4 2017年典型草原两项生态系统服务(功能)散点分布图。 $\mathrm{CK}$, 对照; LG, 轻度放牧; MG, 中度放牧; HG, 重度放牧。 $G E R$, 草地蒸散速率; $G P$, 群落净生长量; $N P R$, 群落净光合 速率; SOC, 土壤有机碳含量; SRR, 土壤呼吸速率; SWC, 土 壤含水量; SWI, Shannon-Wiener指数。

Fig. 4 Scatter plot matrices of paired ecosystem services (functions) in 2017. CK, control; LG, light grazing; MG, moderate grazing; HG, heavy grazing. GER, grassland evapotranspiration rate; $G P$, grassland net growth production; $N P R$, community net photosynthetic rate; SOC, soil organic carbon; $S R R$, soil respiration rate; SWC, soil water content; SWI, Shannon-Wiener index.

\section{3 讨论}

\section{1 放牧对草地生态系统服务和功能的影响}

试验区优势种为大针茅和羊草, 其生物量在调 查的样方生物量中占有绝对优势(大针茅、羊草生物 量占总生物量的 $74.5 \%-97.9 \%)$ 。大针茅属多年生从 生禾草，分藥芽在地下，家畜在采食过程中很难伤 害到分菜芽，放牧初期家畜的采食削弱了顶端效应， 刺激了大针茅的无性分藍过程, 而且随着生长季的 延续, 其粗纤维含量增加, 适口性变差, 逃避采食 及践踏的破碎化使得大针茅获得了更大的生存和繁 殖空间; 羊草属多年生根茎禾草, 群落中羊草与大 针茅混合生长, 种间竞争耗能较少, 放牧强度适中 时, 家畜专注于适口性较好的羊草, 被采食掉多余 的叶片器官和伤口的刺激增加了羊草光资源的补偿 性, 同时放牧减少羊草种间竞争, 刺激羊草的无性 拓殖繁殖, 促进种群间正相互作用的发生(Maestre et al., 2005; 王金金厅等, 2009)。

多样性指数随放牧强度增加显著降低, 这与杨
婧等(2014)的研究结果一致, 生物多样性指数的变 化与中度放牧干扰假说(李永宏, 1993; Joubert et al., 2017)不符。可能是因为放牧虽然削弱了群落层片竞 争，但暴露的下级层片也受到家畜的干扰，造成常 见种和稀有种生物量的下降。同时, 因放牧而降低 高度的植物会加剧地下资源的竞争, 特别是在干旱 年份，导致浅根系和不耐牧植物率先减少和消失， 使植物群落种类单一、结构趋同(Olff et al., 2002)。

土壤含水量作为放牧生态系统一种重要的调节 服务, 与群落地上、地下生物量和调落物量等密切 相关。本实验中放牧显著降低了草地表层土壤含水 量, 是因为植物生物量和调落物量随放牧强度的增
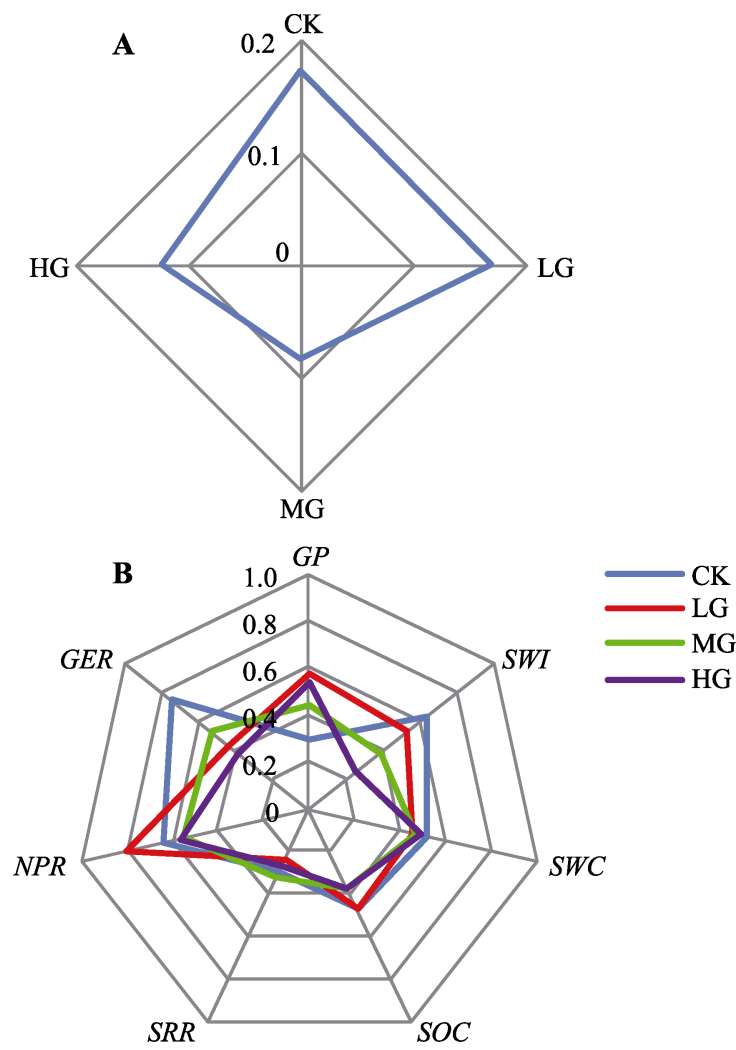

图5 典型草原多项生态系统服务及功能权衡雷达图(A)和 各项服务及功能值雷达图(B)。CK, 对照; $\mathrm{LG}$, 轻度放牧; $\mathrm{MG}$, 中度放牧; HG, 重度放牧。GER, 草地蒸散速率; $G P$, 群落净 生长量; $N P R$, 群落净光合速率; $S O C$, 土壤有机碳含量; $S R R$, 土壤呼吸速率; SWC, 土壤含水量; SWI, Shannon-Wiener指 数。

Fig. 5 Radar plots of trade-offs among multi-ecosystem services (functions)(A) and Radar plots of the ES values (B) of multi-ecosystem services (functions) for the studied grassland under grazing gradients. CK, control; LG, light grazing; MG, moderate grazing; HG, heavy grazing. GER, grassland evapotranspiration rate; $G P$, grassland net growth production; $N P R$, community net photosynthetic rate; SOC, soil organic carbon; SRR, soil respiration rate; SWC, soil water content; SWI, Shannon-Wiener index. 
加而逐渐减少, 对地表径流的拦截作用减弱, 同时 受到的太阳直射越多, 蒸散越强烈。

草地蒸散主要由土壤蒸发和植物蒸腾组成。植 被叶面积指数和地上生物量影响着植物蒸腾在放牧 生态系统蒸散发总量中的贡献比例(Lauenroth \& Bradford, 2006)。植物因被采食增加了创伤面积, 这 些创伤加快了植物自身水分的散失, 加速了植物的 蒸腾速率; 当过度放牧使植物生物量剧烈下降, 参 与草地蒸散过程的植物对蒸散速率的贡献也会大大 降低, 同时, 缺少植物覆盖的地面也会较早地流失 掉土壤中的自由水, 又使植物陷入生理缺水的危机 中。

土壤有机碳含量并不是简单地随放牧强度增加 在下降, 这说明土壤有机碳的变化较地上生物量的 变化具有滞后性, 这与土壤环境条件的稳定性密不 可分。实验中土壤有机碳含量先降后升可能是因为 受放牧行为的影响, 减少了地上植物向地下返还的 有机物，而HG下，被过度采食而失去对地上资源的 竞争优势的物种, 转而增加根系生物量以提高地下 资源的竞争能力, 促使大量的根系残骸转化为土壤 有机物(Gervasio et al., 2010), 且家畜采食和排泄加 速植物的碎裂和调落物的分解, 加快了土壤有机物 的循环; LG和MG植物生产能力越强, 对地上分配 的能量就会越多, 对地下有机物的输送就会不足, 因此土壤有机碳呈先下降后上升的趋势。

本实验中土壤呼吸速率随着放牧强度的增大而 减小。除放牧践踏改变土壤孔隙外, 还与温度和土 壤湿度等条件相关(崔骁勇等, 2000; 李凌浩等, 2000; Wang et al., 2015)。过高的土壤湿度抑制深层 土壤生物的有氧呼吸, 土壤动物和微生物向土壤表 层移动, 土壤化学作用增强, 导致土壤碳排放速率 显著增强; 放牧对草地造成的践踏作用减小土壤孔 隙度, 加剧土壤水分的损失。因此, 不同放牧强度处 理通过改变控制土壤呼吸速率的因子来改变土壤呼 吸速率。

$\mathrm{CK}$ 处理下的群落净光合速率并非最高, 因为 适度放牧剔除植物觉余叶片, 削弱了植物的无序竞 争, 植物叶片参与光合过程的有效叶面积增加, 促 进植物的光补偿能力 (Nie et al., 2004; 晏欣等, 2013)。而CK中过多的叶片不仅没有提高光合速率, 反而增加了过多的被遮阴叶片, 这些圥余的器官又 在不断消耗着植物同化物质(党承林, 1998; 唐海萍
等, 2015)。

\section{2 放牧生态系统多项服务及功能的权衡}

不同服务或功能之间的权衡关系在放牧干扰下 会被削弱, 这与Wu等(2017)在青藏高原不同草地利 用类型的研究相似。这很可能是因为在自然状态下, 植物种间和种内竞争激烈, 对草地资源适度的利用 能削弱植物的竞争作用，提高正相互作用(王金金厅 等, 2015), 另一方面是因为干旱限制了植物的生长, 抑制了植物间的竞争(Austin, 2002); 另一些服务和 功能因放牧而增强了相互间的权衡关系, 可能是因 为放牧行为改变了群落结构, 并反作用于家畜行为, 使群落受干扰的时间和程度发生变化, 进而改变了 群落的理化性质(闰瑞瑞等, 2009; Onatibia et al., 2017)。其他一些服务功能间的关系并没有因放牧强 度不同而发生明显变化, 这主要发生在土壤有机碳 含量与其他服务和功能间的权衡关系中, 因统计学 原因和自然属性因素的关系, 土壤有机碳含量在放 牧梯度下无显著差异, 意味着土壤有机碳的误差值 较低, 极大值、极小值与均值的偏离程度低, 所得的 各放牧强度下生态系统服务值接近, 而土壤本身的 稳定性和时滞性使它和其他生态系统服务及功能间 的关系保持相对稳定。

在连续5年放牧后, CK、LG、MG和HG的土壤 有机碳含量下降了 $14.7 \%-21.4 \%$ (2012年在放牧前 对4种放牧强度土壤样品进行化验分析, 土壤有机 碳含量分别为 $18.7 、 16.34 、 16.18$ 和 $17.82 \mathrm{~g} \cdot \mathrm{kg}^{-1}$ ), 这 可能是因为长期围封下植物碳(C) 以调落物形式积 存在土壤表面, 使植物 $\mathrm{C}$ 转化为土壤 $\mathrm{C}$ 的过程变得 更加缓慢(苏永中和赵哈林, 2003); 三种放牧管理中, 植物除了变成调落物向土壤中返还, 还有一部分被 家畜采食无法返还到土壤中, 土壤有机碳在供给植 物生长和调落物返还的循环中一直处于亏损状态, 表现为土壤碳库与植物碳库的正相关关系(刘忠宽 等, 2006)。有研究表明, 土壤有机碳虽然未直接影 响生物多样性, 却能促进根际微生物碳源的输入和 土壤氮矿化效率从而间接影响生物多样性(Cong et al., 2015)。长期放牧对土壤C库的影响也打破了土 壤微生物和土壤C固持、排放的收支平衡, 改变了土 壤呼吸速率。因此, 土壤有机碳含量通过改变其他 服务和功能进而影响着其生态系统服务和功能间的 权衡关系。

多项生态系统服务及功能权衡中, 最适的放牧 
强度并不是按照各单项服务及功能在不同放牧强度 下发挥最大服务能力，也不是按照任意两项服务或 功能发生协同来判定, 而是要依据草地生态系统整 体的协调性。本实验的多项生态系统服务和功能权 衡中, $\mathrm{CK}$ 的权衡关系最强, MG放牧的权衡关系最弱, 很可能是由生态系统中 “木桶效应” 引起的 $(\mathrm{He} \&$ Sun, 2015)。标准化后的多项服务和功能权衡的草地 生态系统中, CK处理下的群落净生长量值最低, LG 处理中土壤呼吸速率值最低, HG处理中生物多样性 和草地蒸散速率值最低, 因此, 各放牧强度下的草 地生态系统协同性就是由服务功能最弱的那项“短 板”服务或功能所决定的(图5B)。因此, 从多项服务 和功能权衡的角度评价草地生态系统协调性, 需要 通过合理的放牧强度, 合理使用草地资源, 避免出 现草地生态系统服务及功能“短板”。

大尺度区域的生态系统服务及功能权衡决策管 理, 常引入公民意见, 使决策意见更易被理解和接 受(Brown et al., 2001; Karjalainen et al., 2013)。但在 中小尺度区域进行生态系统服务及功能权衡时, 这 种权威性的办法反而显得臃肿, 如权衡周期过长, 经济代价增大, 以及各统计指标物质量单位不统一 等，同时权衡过程也会受到权衡参与者教育程度、 个人偏好、职业等主观因素的影响(王举位等, 2013)。目前, 使用极值法对测量指标进行无量纲化 处理可以克服物质量评估的量纲单位不统一问题, 同时规避了赋权评价对生态系统服务及功能权衡关 系的主观影响, 在实际应用中较为广泛、灵活; 统 计变量 $R M S D$ 便于计算, 并且不受时间尺度和空间 尺度的影响，权衡方法简单客观(Berta et al., 2012; Bradford \& D'Amato, 2012)。

\section{4 结论}

在放牧管理的草地生态系统服务和功能的权 衡中, 权衡、协同和不相关关系同时存在, 如土壤呼 吸速率与群落净生长量、多样性与群落净光合速率 存在权衡关系; 群落净生长量分别与土壤含水量、 群落净光合速率、草地蒸散速率及生物多样性, 土 壤呼吸速率分别与草地蒸散速率及生物多样性, 这 些服务(功能)间存在协同关系。从长期实验来看, 土 壤有机碳含量由于放牧等管理方式的改变应该发生 较大变化, 这对整体草地生态系统服务的支持功能 有重要影响, 也相应影响着其服务间的权衡和协
同。放牧能不同程度地削弱多项生态系统服务及功 能的权衡关系(冲突关系); 从多项生态系统服务和 功能权衡的观点比较最适放牧强度, 中度放牧的生 态系统服务和功能协同关系最佳(冲突最小)。

致谢 感谢中国气象局锡林浩特国家气象观象台的 帮助和项目基金的支持。

\section{参考文献}

Austin AT (2002). Differential effects of precipitation on production and decomposition along a rainfall gradient in Hawaii. Ecology, 83, 328-338.

Austrheim G, Speed JDM, Evju M, Holand AHO, Loe LE, Martinsen V, Mobak R, Mulder J, Steen H, Thompson DBA, Mysterud A (2016). Synergies and trade-offs between ecosystem services in an alpine ecosystem grazed by sheep-An experimental approach. Basic and Applied Ecology, 17, 596-608.

Bai YF, Huang JH, Zheng SX, Pan QM, Zhang LX, Zhou HK, $\mathrm{Xu}$ HL, Li YL, Ma J (2014). Drivers and regulating mechanisms of grassland and desert ecosystem services. Chinese Journal of Plant Ecology, 38, 93-102. [白永飞, 黄建辉, 郑淑霞, 潘庆民, 张丽霞, 周华坤, 徐海量, 李 玉霖, 马健 (2014). 草地和荒漠生态系统服务功能的形 成与调控机制. 植物生态学报, 38, 93-102.]

Berta ML, Irene IA, Martain Garcia L, Ignacio P, Izaskun CA, David GDA, Erik GB, Elisa OR, Igone PA, Barbara W, Jose AG, Fernando SM, Miren O, Cesar LS, Carlos M (2012). Uncovering ecosystem service bundles through social preferences. PLOS ONE, 7, e38970. DOI: 10.1371/ journal.pone.0038970.

Bradford JB, D'Amato AW (2012). Recognizing trade-offs in multi-objective land management. Frontiers in Ecology and the Environment, 10, 210-216.

Brown K, Adger WN, Tompkins E, Bacon P, Shim D, Young K (2001). Trade-off analysis for marine protected area management. Ecological Economics, 37, 417-434.

Cong WF, Jasper VR, Liesje M, Gerlinde BDD, Frank B, Ellis $\mathrm{H}$ (2015). Plant species richness promotes soil carbon and nitrogen stocks in grasslands without legumes. Journal of Ecology, 102, 1163-1170.

Cui XY, Chen SQ, Chen ZZ (2000). $\mathrm{CO}_{2}$ release from typical Stipa grandis grassland soil. Chinese Journal of Applied Ecology, 11, 390-394. [崔骁勇, 陈四清, 陈佐忠 (2000). 大针茅典型草原土壤 $\mathrm{CO}_{2}$ 排放规律的研究. 应用生态学 报, 11, 390-394.]

Dang CL (1998). Redundancy structure in phytocoenosiums as an explanation of ecosystem stability. Acta Ecologica Sinica，18，665-672. [党承林 (1998). 植物群落的午余 结构——对生态系统稳定性的一种解释. 生态学报, 18 , 
665-672.]

Davidson KE, Fowler MS, Skov MW, Doerr SH, Beaumont N, Griffin JN, Bennett J (2017). Livestock grazing alters multiple ecosystem properties and services in salt marshes: A meta-analysis. Journal of Applied Ecology, 54, 1365-1401.

Fu BJ, Zhou GY, Bai YF, Song CC, Liu JY, Zhang HY, Lü YH, Zheng H, Xie GD (2009). The main terrestrial ecosystem services and ecological security in China. Advances in Earth Science, 24, 571-576. [傅伯杰, 周国逸, 白永飞, 宋长春, 刘纪远, 张惠远, 吕一河, 郑华, 谢高 地 (2009). 中国主要陆地生态系统服务功能与生态安 全. 地球科学进展, 24, 571-576.]

Gervasio P, Jose MP, Martin O, Esteban GJ (2010). Pathways of grazing effects on soil organic carbon and nitrogen. Rangeland Ecology \& Managemengt, 63, 109-119.

Han GD, Li B, Wei ZJ, Yang J, Lü X, Li H (1999). Plant compensatory growth in the grazing system of Stipa breviflora desert steppe: I. Plant net productivity. Acta Agrestia Sinica，7(1)，1-7. [韩国栋, 李博, 卫智军, 杨静, 吕雄, 李宏 (1999). 短花针茅草原放牧系统植物补偿性生长 的研究: I. 植物净生长量. 草地学报, 7(1), 1-7.]

He JL, Sun YR (2015). Applying a spatial decision support system to the integrated regional planning of China. Environment and Planning B Planning and Design, 47, 1-16.

Hector A, Bagchi R (2007). Biodiversity and ecosystem multifunctionality. Nature, 448, 188-190.

Joubert L, Pryke JS, Samways MJ (2017). Moderate grazing sustains plant diversity in Afromontane grassland. Applied Vegetation Science, 20, 340-351.

Karjalainen TP, Marttunen M, Sarkki S, Rytkönen AM (2013). Integrating ecosystem services into environmental impact assessment: An analytic-deliberative approach. Environmental Impact Assessment Review, 40, 54-64.

Lauenroth WK, Bradford JB (2006). Ecohydrology and the partitioning AET between transpiration and evaporation in a semiarid steppe. Ecosystems, 9, 756-767.

Li LH, Wang QB, Bai YF, Zhou GS, Xing XR (2000). Soil respiration of a Leymus chinensis grassland stand in the Xilin River Basin as affected by over-grazing and climate. Acta Phytoecologica Sinica, 24, 680-686. [李凌浩, 王 其兵, 白永飞, 周广胜, 邢雪荣 (2000). 锡林河流域羊 草草原群落土壤呼吸及其影响因子的研究. 植物生态 学报, 24, 680-686.]

Li YH (1993). Grazing dynamics of the species diversity in Aneurolepidium chinense steppe and Stipa grandis steppe. Acta Botanica Sinica, 35, 877-884. [李永宏 (1993). 放 牧影响下羊草草原和大针茅草原植物多样性的变化. 植物学报, 35, 877-884.]

Liang MW, Liang CZ, Bai X, Miao BL, Wang YS, Bao GR, Wang X (2016). Effects of annual plant functional group on biomass and soil respiration in a grazing community of a typical steppe grassland. Prataculitural Science, 33, 2407-2417. [梁茂伟, 梁存柱, 白雪, 苗百岭, 王英舜,
包桂荣，王譞 (2016). 一年生植物功能群对放牧草原生 物量和土壤呼吸的影响. 草业科学, 33, 2407-2417.]

Liu ZK, Wang SP, Chen ZZ, Wang YF, Han JG (2006). Properties of soil nutrients and plant community after rest grazing in Inner Mongolia steppe, China. Acta Ecologica Sinica，26，2048-2056. [刘忠宽，汪诗平，陈佐忠，王艳 芬, 韩建国 (2006). 不同放牧强度草原休牧后土壤养分 和植物群落变化特征. 生态学报, 26, 2048-2056.]

Lu N, Fu BJ, Jin TT, Chang RY (2014). Trade-off analyses of multiple ecosystem services by plantations along a precipitation gradient across Loess Plateau landscapes. Landscape Ecology, 29, 1697-1708.

Maestre FT, Valladares F, Reynolds JF (2005). Is the change of plant-plant interactions with abiotic stress predictable? A meta-analysis of field results in arid environments. Journal of Ecology, 93, 748-757.

Mcintyre S, Heard M, Martin TG (2003). The relative importance of cattle grazing in subtropical grasslands: Does it reduce or enhance plant biodiversity? Journal of Applied Ecology, 40, 445-457.

Millennium Ecosystem Assessment (2005). Ecosystem and Human Well-being. Island Press, Washington DC.

Naidoo R, Balmford A, Costanza R, Fisher B, Green RE, Lehner B, Malcolm TR, Ricketts TH (2008). Global mapping of ecosystem services and conservation priorities. Proceedings of the National Academy of Sciences of the United States of America, 105, 9495-9500.

Nie ZN, Chapman DF, Tharmaraj J, Clements R (2004). Effects of pasture species mixture, management, and environment on the productivity and persistence of dairy pastures in south-west Victoria. 1. Herbage accumulation and seasonal growth pattern. Australian Journal of Agricultural Research, 55, 625-636.

Odum E (1971). Fundamentals of Ecology. Philadelphia, Saunders.

Olff H, Ritchie ME, Prins HHT (2002). Global environmental controls of diversity in large herbivores. Nature, 415, 901-904.

Onatibia GR, Reyes MF, Aguiar MR (2017). Fine-scale root community structure and below-ground responses to grazing show independence from above-ground patterns. Journal of Vegetation Science, 28, 1097-1106.

Pan Y, Wu JX, Xu ZR (2014). Analysis of the tradeoffs between provisioning and regulating services from the perspective of varied share of net primary production in an alpine grassland ecosystem. Ecological Complexity, 17, 79-86.

Pan Y, Xu ZR, Wu JX (2013). Spatial differences of the supply of multiple ecosystem services and the environmental and land use factors affecting them. Ecosystem Services, 5, 4-10.

Pucheta E, Bonamici I, Cabido M, Diaz S (2010). Belowground biomass and productivity of a grazed site and a

www.plant-ecology.com 
neighbouring ungrazed exclosure in a grassland in central Argentina. Austral Ecology, 29, 201-208.

Rodríguez JP, Beard Jr TD, Bennett EM, Cumming GS, Cork S, Agard J, Dobson AP, Peterson GD (2006). Trade-offs across space, time, and ecosystem services. Ecology and Society, 11, 28-41.

$\mathrm{Su}$ YZ, Zhao HL (2003). Influences of grazing and exclosure on carbon sequestration in degraded sandy grassland, Inner Mongolia, North China. Environmental Science, 24(4), 23-28. [苏永中, 赵哈林 (2003). 持续放牧和围封对科 尔沁退化沙地草地碳截存的影响. 环境科学, 24(4), 23-28.]

Tang HP, Xue HL, Fang F (2015). A comparison of measured and calculated net community $\mathrm{CO}_{2}$ exchange: Scaling from leaves to communities. Chinese Journal of Plant Ecology, 39, 924-931. [唐海萍, 薛海丽, 房飞 (2015). 叶片和群落尺度净光合速率关系的探讨. 植物生态学 报, 39, 924-931.]

Wang D, Wu GL, Liu Y, Yang Z, Hao HM (2015). Effects of grazing exclusion on $\mathrm{CO}_{2}$ fluxes in a steppe grassland on the Loess Plateau (China). Ecological Engineering, 83, $169-175$.

Wang DL, Wang L (2019). A new perspective on the concept of grassland management. Chinese Science Bulletin, 64, 1106-1113. [王德利, 王玲 (2019). 草地管理概念的新 释义. 科学通报, 64, 1106-1113.]

Wang JW, Zhang Z, An BL, Yan GZ (2013). Index weights for seabuckthorn plantation ecosystem services evaluation in the soft rock area. Journal of Arid Land Resources and Environment, 27(10), 145-151. [王举位, 张征, 安宝利, 间国振 (2013). 砒砂岩区沙棘人工林生态系统服务功 能评价指标权重初探. 干旱区资源与环境，27(10), 145-151.]

Wang L, Manuel DB, Wang DL, Forest I, Liu J, Feng C, Liu JS, Zhong ZW, Zhu H, Yuan X, Chang Q, Liu C (2019). Diversifying livestock promotes multidiversity and multifunctionality in managed grasslands. Proceedings of the National Academy of Sciences of the United States of America, 116, 6187-6192.

Wang SP, Li YH, Chen ZZ (1999). The optimal stocking rates on grazing system in Inner Mongolia steppe: I. Based on analysis of liveweight gain per animal and hectare and benefit. Acta Agrestia Sinica, 7(3), 183-191. [汪诗平, 李永宏, 陈佐忠 (1999). 内蒙古典型草原草畜系统适宜 放牧率的研究: I. 以绵羊增重及经济效益为管理目标. 草地学报, 7(3), 183-191.]

Wang XT, Wang W, Liang CZ (2009). Changes in the population spatial distribution pattern of Leymus chinensis in degraded steppe community during restorative succession in Inner Mongolia, China. Chinese Journal of Plant Ecology, 33, 63-70. [王釒鍂厅, 王炜, 梁存柱 (2009). 典型草原退
化群落不同恢复演替阶段羊草种群空间格局的比较. 植物生态学报, 33, 63-70.]

Wang XT, Wang W, Liang CZ, Liu ZL (2015). Interpretation of grassland degradation caused by overgrazing from the perspective of positive interaction. Chinese Science Bulletin, 60, 2794-2799. [王金锤厅, 王炜, 梁存柱, 刘钟龄 (2015). 从正相互作用角度诠释过度放牧引起的草原退 化. 科学通报, 60, 2794-2799.]

Wu JX, Zhao Y, Yu CQ, Luo LM, Pan Y (2017). Land management influences trade-offs and the total supply of ecosystem services in alpine grassland in Tibet, China. Journal of Environmental Management, 193, 70-78.

Xu W, Jing X, Ma ZY, He JS (2016). A review on the measurement of ecosystem multifunctionality. Biodiversity Science, 24, 72-84. [徐炜, 井新, 马志远, 贺金生 (2016). 生态系统多功能性的测度方法. 生物多样性, 24, 72-84.]

Xue R, Zheng SX, Bai YF (2010). Impacts of grazing intensity and management regimes on aboveground primary productivity and compensatory growth of grassland ecosystems in Inner Mongolia. Biodiversity Science, 18, 300-311. [薛睿，郑淑霞，白永飞 (2010). 不同利用方 式和载畜率对内蒙古典型草原群落初级生产力和植物 补偿性生长的影响. 生物多样性, 18, 300-311.]

Yan RR, Wei ZJ, Yun XJ, Chu WB, WQ, Xin XP (2009). Effects of the grazing systems on diurnal variation of photosynthetic characteristic of major plant species of desert steppe. Acta Prataculturae Sinica, 18(5), 160-167. [阊瑞 瑞, 卫智军, 运向军, 褚文彬, 乌仁其其格, 辛晓平 (2009). 放牧制度对短花针茅荒漠草原主要植物种光合 特性日变化影响的研究. 草业学报, 18(5), 160-167.]

Yan X, Gong JR, Zhang ZY, Huang YM, An R, Qi Y, Liu M (2013). Responses of photosynthetic characteristics of Stipa baicalensis to grazing disturbance. Chinese Journal of Plant Ecology, 37, 530-541. [晏欣, 龚吉芯, 张梓瑜, 黄永梅, 安然, 祁瑜, 刘敏 (2013). 狼针草光合特性对 放牧干扰的响应. 植物生态学报, 37, 530-541.]

Yang J, Chu PF, Chen DM, Wang MJ, Bai YF (2014). Mechanisms underlying the impacts of grazing on plant $\alpha, \beta$ and $\gamma$ diversity in a typical steppe of the Inner Mongolia grassland. Chinese Journal of Plant Ecology, 38, 188-200. [杨 婧, 褚鹏飞, 陈迪马, 王明玖, 白永飞 (2014). 放牧对 内蒙古典型草原 $\alpha 、 \beta$ 和 $\gamma$ 多样性的影响机制. 植物生态 学报, 38, 188-200.]

Zavaleta ES, Pasari JR, Hulvey KB, Tilman GD (2010). Sustaining multiple ecosystem functions in grassland communities requires higher biodiversity. Proceedings of the $\mathrm{Na}$ tional Academy of Sciences of the United States of America, 107, 1443-1446.

责任编委: 王德利 责任编辑: 李 敏 实习编辑: 赵 航 